\title{
Throughput Characterization for Cooperative Wireless Information Transmission with RF Energy Harvesting-Based Relay
}

\author{
Yuanyuan Yao, ${ }^{1}$ Changchuan Yin, ${ }^{1}$ and Sai Huang ${ }^{2}$ \\ ${ }^{1}$ Beijing Laboratory of Advanced Information Networks and the Beijing Key Laboratory of Network System Architecture and \\ Convergence, Beijing University of Posts and Telecommunications, Beijing 100876, China \\ ${ }^{2}$ Key Laboratory of Universal Wireless Communications Ministry of Education, Wireless Technology Innovation Institute (WTI), \\ Beijing University of Posts and Telecommunications, Beijing 100876, China
}

Correspondence should be addressed to Yuanyuan Yao; yyyao@bupt.edu.cn

Received 25 January 2016; Accepted 27 March 2016

Academic Editor: Yunfei Chen

Copyright (C) 2016 Yuanyuan Yao et al. This is an open access article distributed under the Creative Commons Attribution License, which permits unrestricted use, distribution, and reproduction in any medium, provided the original work is properly cited.

\begin{abstract}
The simultaneous wireless information and power transfer (SWIPT) in a cooperative relaying system is investigated, where the relay node is self-sustained by harvesting radiofrequency (RF) energy from the source node. In this paper, we propose a time switching and power splitting (TSPS) protocol for the cooperative system with a mobile destination node. In the first part of the transmission slot, a portion of the received signal power is used for energy transfer, and the remaining power is used for information transmission from the source to the relay. For the remaining time of the transmission slot, information is transmitted from the relay to a mobile destination node. To coordinate the wireless information and power transfer, two transmission modes are investigated, namely, relay-assisted transmission mode and nonrelay mode, respectively. Under these two modes, the outage probability and the network throughput are characterized. By joint optimization of the power splitting and the time switching ratios, we further compare the network throughput under the two transmission modes with different parameters. Results indicate that the relayassisted transmission mode significantly improves the throughput of the wireless network.
\end{abstract}

\section{Introduction}

Recently, to prolong the lifetime of energy-constrained wireless network, energy harvesting has drawn great attentions due to the growing energy demands and the increasing energy prices [1]. It enables the wireless nodes to collect energy from ambient renewable energy sources (e.g., wind and solar power), which eliminates the need for manual battery replacement/recharging, and effectively reduces the cost of energy bills and degrades the level of carbon dioxide emissions [2]. However, for some devices, such as military nodes or wireless sensor nodes which are located in a harsh environment that is difficult to access, the recharging of the batteries remains an open problem, especially when the number of nodes is huge and the nodes are distributed in a wide area. Radiofrequency (RF) energy harvesting is proposed as a solution or partial solution to overcome these problems [3].

$\mathrm{RF}$ energy harvesting can be regarded as a far-field energy transfer technique and has become a promising solution for generating a small amount of electrical power to replenish the power sources in energy-constrained wireless networks $[4,5]$. The RF harvester in the node is equipped with a power conversion circuit, which can transform the received electromagnetic wave into direct-current (DC) power. As such, the devices can utilize the harvested energy from RF signals to augment/replenish their batteries [6]. Wireless nodes which are equipped with omnidirectional antennas can radiate RF signals in all directions, and thus the wireless signals can be used to deliver information as well as energy, which indicates that the energy-constrained wireless devices can simultaneously process wireless information and 
power transfer (SWIPT) [7]. With SWIPT, the available wireless resources can be effectively utilized by developing the transceiver designs.

The idea of SWIPT was first proposed in [8], where a capacity-energy function was utilized to investigate the tradeoff between energy harvesting and information transmission. Since the traditional receiver cannot extract the RF energy from the same signals used for information transmission, a new SWIPT receiver that can split the received signal into two separate streams was proposed in [9], where the two streams can be used for information decoding and energy harvesting with different power levels. Liu et al. in [10] derived the optimal information decoding and energy harvesting mode switching rules at the receiver targeting the optimization of the outage probability by using time switching (TS) or power splitting (PS) protocols. In [11], Ng et al. investigated the resource allocation algorithm that is designed to maximize the energy efficiency of data transmission in orthogonal frequency division multiple access (OFDMA) systems with SWIPT, and suboptimal iterative resource allocation algorithms were formulated and solved.

The cooperative relaying techniques can overcome the fading and the attenuation by using the intermediate relay, which significantly improve the efficiency and reliability of the network. Therefore, it is appropriate to be used in energy-constrained networks such as the RF energy harvesting networks [7]. Relaying cooperation is integrated in some standards and systems for providing different levels of assistance. In [12], PS-based relaying (PSR) protocol and the TS-based relaying (TSR) protocol were investigated to enable information decoding and energy harvesting at the energy-constrained relay in wireless amplify-and-forward (AF) relaying networks. In the PSR protocol, the relay used part of the received signal power for energy harvesting and the remaining signal power for information transmission. In the TSR protocol, the relay spent a portion of time for energy harvesting and the remaining time for information transmission. The SWIPT in an OFDM relaying system was considered in [13], where a source node transmitted energy and information simultaneously to a relay, and then the relay forwards the source information by using the harvested energy to the destination node. However, in $[12,13]$, some issues need to be further addressed: (a) the network throughput is analyzed separately in terms of power splitting ratio or time switching ratio, and joint optimization in terms of the two parameters is never considered; (b) in the cooperative relaying system, the location of the relay or the distances between nodes are restricted; for example, in [12], the authors assume that the distance between the relay and the destination node is a constant, and, in [13], the relay is located on a line between the source and the destination; (c) most of the prior works assumed that there is no direct link between the source and the destination node in the cooperative relaying system, and thus we could not evaluate whether the performance is improved by exploiting the cooperative relaying concept with energy harvesting.

In this paper, as shown in Figure 1, we consider a threenode cooperative relaying system, where two transmission modes are investigated, that is, (a) the nonrelay mode, where

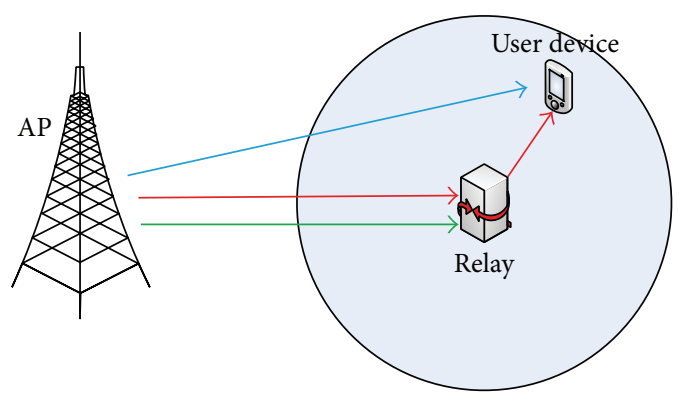

$$
\begin{aligned}
& \text { Nonrelay mode } \longrightarrow \text { Information flow } \\
& \text { Relay-assisted mode } \longrightarrow \text { Information flow } \\
& \text { Transmission mode } \longrightarrow \text { Energy flow }
\end{aligned}
$$

FIgURE 1: System model for RF energy harvesting-based relay network.

the access-point (AP) directly transmits the information to the user device (UD), no matter when the UD is far away from the AP or not, and (b) the relay-assisted transmission mode, where the AP transmits the information directly to the UD when the UD is relatively nearer to the AP; for the UD located far away from the AP, an energy harvesting relay assists the message transmission from the source to the UD [14]. Time is partitioned into slots. For the relay-assisted transmission mode, a time switching and power splitting (TSPS) protocol for the relaying path is proposed. In each transmission slot, during the first part of the slot, a portion of the signal power in the source is used for energy transfer, and the remaining power is used for information transmission from the source to the relay; for the remaining transmission time, information is transmitted from the relay to the destination. The position of the destination node is assumed to be changed uniformly within a circular area on $R^{2}$ around the relay from slot to slot. The main contributions of the paper are summarized as follows. (1) We analyze the performance for cooperative relaying system with a mobile destination, assuming that the position of the destination node uniformly changes from slot to slot. (2) A function describing the relationship between the network performance (i.e., the outage probability and the network throughput) and both the power splitting and the time switching ratios is given. (3) We evaluate and compare the performance for the two transmission modes with different parameters, and the relay-assisted mode considerably improves the reliability of the wireless network.

The remainder of this paper is organized as follows. The system model and performance metrics are described in Section 2. Section 3 investigates the network throughput with the relay-assisted transmission mode. The network throughput with nonrelay mode is studied in Section 4. Numerical results are presented in Section 5. Finally, we conclude the paper in Section 6 .

Notations. Throughout this paper, we use $E[\cdot]$ and $|\cdot|$ to denote the expectation operator and the absolute value operations, respectively. $X \sim \mathscr{C} \mathcal{N}\left(\mu, \sigma^{2}\right)$ stands for a circularly symmetric complex Gaussian random variable $X$ 


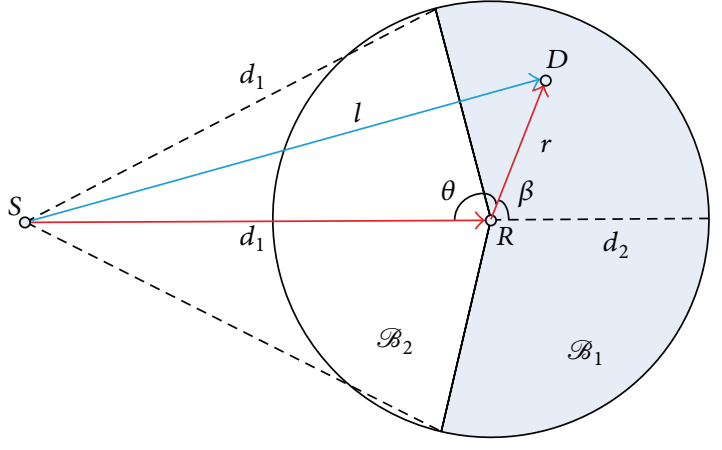

FIGURE 2: System topology for energy-constrained relay-assisted network.

with mean $\mu$ and variance $\sigma^{2}$, while $X \sim \exp (\lambda)$ represents an exponentially distributed random variable $X$ with mean $\lambda$.

\section{System Model}

2.1. Network Model. We consider a wireless communication network which consists of one single-antenna AP named source $S$, one user device named destination node $D$, and another terminal considered as an energy-constrained relay, denoted by $R$. As shown in Figure 2, the information is transmitted from $S$ to $D$, with/without $R$. It is assumed that the source is continuously connected to a power supply and the transmission power is $P_{s}$; the relay has no direct power supply but is embedded with a rechargeable battery and thus could harvest energy from the RF signal broadcasted by the source. In addition, the relay has no traffic and is dedicated to help the source forward the information from source to destination. Furthermore, we assume the relay receives and transmits signals over two different frequency bands.

The propagation channel is modeled as the combination of small-scale Rayleigh fading and large-scale path loss given by

$$
h_{X Y}=\widetilde{h}_{X Y} \sqrt{r_{X Y}^{-\alpha}}
$$

where $\widetilde{h}_{X Y} \sim \mathscr{C} \mathcal{N}\left(0, \mu_{X Y}\right)$ denotes the channel coefficients from $X$ to $Y$ with $X, Y \in\{S, R, D\}$ and $X \neq Y$, the channel power gain $\left|\widetilde{h}_{X Y}\right|^{2}$ follows the exponential distribution with the mean $\mu_{X Y}$, that is, $\left|\widetilde{h}_{X Y}\right|^{2} \sim \exp \left(\mu_{X Y}\right), r_{X Y}$ denotes the propagation distance from $X$ to $Y$, and $\alpha>2$ is the path-loss exponent.

Time is partitioned into slots with duration $T$. During each slot, the channel gains remain constant but are independently and identically distributed (i.i.d.) from one slot to another. The distance between the source and the relay is $d_{1}$; that is, $r_{S R}=d_{1}$. We assume that, within a time slot, the position of the destination node uniformly changes from slot to slot within the transmitting area (available region) of the relay, named $\mathscr{B}$, which denotes a disk centered at $R$ with radius $d_{2}$. Therefore, the probability density function (PDF) of the distance from the relay to the destination $r_{R D}$ is given by $f_{r_{R D}}(r)=2 r / d_{2}^{2}$.
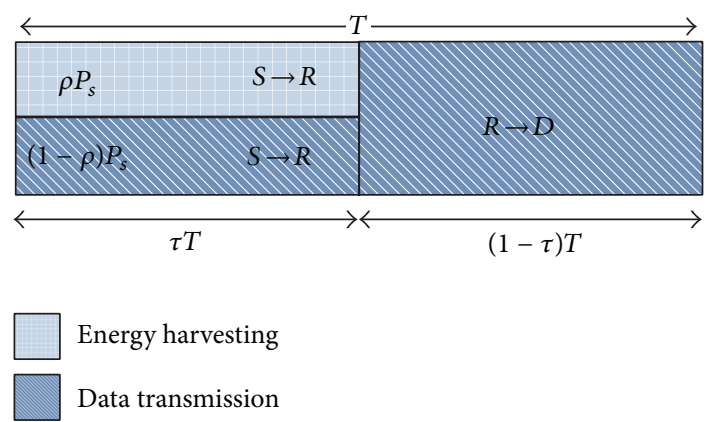

FIGURE 3: Transmission slot structure for TSPS protocol.

2.2. Selection Sector Model. The considered network does not require the source to choose the relaying path all the time. Provided that the $S-D$ link is poor and the destination node is far away from the source, the relay can help deliver the source signals to the destination in order to combat fading and pathloss degradation effects. However, when the destination node is relatively nearer to the source, choosing the $S-R-D$ path can not only lead to a waste of resources but also limit the network capacity. Therefore, we define two complementary sectors $\mathscr{B}_{1}$ and $\mathscr{B}_{2}$, as shown in Figure 2, which have a significant influence on the network performance.

We define $\theta \triangleq \angle S R D$ as the angle which is formed by the source $S$, the relay $R$, and the destination node $D$. In addition, we use subscript $l$ for $r_{S D}$ and use subscript $r$ for $r_{R D}$. We define the area $\mathscr{B}_{2}$ as $\mathscr{B}_{2}=\left\{r \in\left[0, d_{2}\right], \theta \in\left[-\theta_{0}, \theta_{0}\right]\right\}$, with $\theta_{0}=\arccos \left(d_{2} / 2 d_{1}\right)$. By using the cosine rule, the sourcerelay distance is given by $l=\sqrt{d_{1}^{2}+r^{2}-2 d_{1} r \cos \theta}$.

Then, we define $\beta=\pi-\theta$ as the center angle of the shaded area $\mathscr{B}_{1}$; the selection region is denoted by $\mathscr{B}_{1}=\{r \in$ $\left.\left[0, d_{2}\right], \beta \in\left[-\beta_{0}, \beta_{0}\right]\right\}$, with $\beta_{0}=\pi-\arccos \left(d_{2} / 2 d_{1}\right)$. It is worth noting that $\beta_{0}$ is the maximum range of the angle.

The remaining area $\mathscr{B}_{2}$ is the direct-link area, which means that the relay is ineffective if the destination node $D$ is inside this area.

2.3. Transmission Mode. To analyze the system performance, we consider two transmission modes: (a) the nonrelay mode and (b) the relay-assisted transmission mode, respectively. In mode (a), the source directly transfers the information to the destination, no matter when the destination node is far away from the source or not. However, in mode (b), the source only transmits the information directly to the destination during the whole time when the destination node is relatively nearer to the source than the relay, that is, the destination node located inside the area $\mathscr{B}_{2}$. When the destination node is located inside the area $\mathscr{B}_{1}$, the cooperative relaying concept is exploited; a TSPS protocol for the relaying path is proposed; as depicted in Figure 3, in each transmission slot, denoted by $T$, during the first part of the time $\tau T$, where $\tau$ is the time switching ratio with $0<\tau<1$, a portion of the transmission power, $\rho P_{s}$, is used for energy transfer from the source to the relay, and the remaining power, $(1-\rho) P_{s}$, is used for information transmission from the source to the relay, where 
$\rho$ is the power splitting ratio $0<\rho<1$. The remaining part of the time, $(1-\tau) T$, is used for information transmission from relay to destination.

Note that we assume the circuit power (i.e., the power consumed by the hardware during data transmission) is negligible; therefore, all the energy harvested from the source is consumed by the relay when the information is delivered from the relay to the destination. The values of the time fraction $\tau$ and the power fraction $\rho$, used for wireless information and energy transfer by the relay, have a significant impact on the achievable throughput at the destination.

The notations and symbols used in the paper are listed in Symbol Notation.

2.4. Performance Metric. In this paper, two performance metrics are studied, which are defined as follows.

2.4.1. Outage Probability. The outage probability is defined as the probability that a receiver decodes the received data packets unsuccessfully from its corresponding transmitter. Specifically, given the signal-to-noise ratio (SNR) and a corresponding SNR target, denoted by $\gamma$, the outage probability of the network is defined as

$$
p_{\text {out }}=\mathbb{P}(\mathrm{SNR}<\gamma) .
$$

2.4.2. Network Throughput. The network throughput is the maximum rate that the system can achieve with successful transmissions. Assume that the source transmission rate target is $R_{r}=\log (1+\gamma)$, and the total transmission time is $t$. Consequently, the network throughput is given by

$$
C=\left(1-p_{\text {out }}\right) \cdot R_{r} \cdot t
$$

\section{Relay-Assisted Transmission Mode}

In this mode, as shown in Figure 2, the transmitting area of the relay $\mathscr{B}$ is split into two parts, the relay selection sector $\mathscr{B}_{1}$ for the relay path $S-R-D$ and direct-link area $\mathscr{B}_{2}$ for the path $S$ - $D$. In this section, we first derive the outage probability based on the TSPS protocol for the $S-R-D$ link and the direct link $S-D$, respectively. Then, we characterize the average network throughput by considering different locations of the destination node.

3.1. Cooperative Link $(S-R-D)$. As shown in Figure 2, the probability that the destination node $D$ is located inside the area $\mathscr{B}_{1}$ is derived as

$$
p_{1}=\frac{1}{2 \pi} \int_{-\beta_{0}}^{\beta_{0}} \mathrm{~d} \beta=\frac{\pi-\arccos \left(d_{2} / 2 d_{1}\right)}{\pi} .
$$

Note that $p_{1}$ increases with $d_{2}$ and decreases with $d_{1}$.

During the first part of the transmission slot with duration $\tau T$, the received signal from the source by the relay, denoted by $y_{r}$, is split into two parts by a ratio $\rho$. The first part $\sqrt{\rho} y_{r}$ is sent to the energy harvesting receiver, which can collect RF signal directly and transform it into DC power, while the remaining part $\sqrt{1-\rho} y_{r}$ is sent to the information receiver. We have

$$
y_{r}=\sqrt{P_{s}} h_{S R} \cdot s(m)+n_{a}
$$

where $h_{S R}=\tilde{h}_{S R} \sqrt{d_{1}^{-\alpha}}, s(m)$ is the normalized transmitted signal from the source, $\mathbb{E}\left\{|s(m)|^{2}\right\}=1$, and $n_{a}$ is the additive white Gaussian noise (AWGN) caused by the receiving antenna of the relay. Then, the energy harvested by the relay from the source is given by

$$
E_{h}=\eta \rho P_{s}\left|h_{S R}\right|^{2} \tau T
$$

where $\eta(0<\eta \leq 1)$ denotes the harvesting efficiency.

The information receiver transforms the received RF signal to baseband and accomplishes baseband signal processing, and then the sampled signal, $y_{r}(m)=\sqrt{1-\rho} y_{r}$, at the relay is obtained as

$$
y_{r}(m)=\sqrt{(1-\rho) P_{s}} h_{S R} \cdot s(m)+\sqrt{(1-\rho)} n_{a}+n_{b}
$$

where $n_{b}$ is the AWGN caused by baseband signal processing.

For the remaining part of the time slot, $(1-\tau) T$, the relay will amplify and forward the sampled signal to the destination using the power $P_{r}$, which is given by

$$
P_{r}=\frac{E_{h}}{(1-\tau) T}=\frac{\eta \rho P_{s}\left|h_{S R}\right|^{2} \tau}{(1-\tau)} .
$$

The amplification factor is given by [15]

$$
\xi=\frac{1}{\sqrt{A}}=\frac{1}{\sqrt{(1-\rho) P_{s}\left|h_{S R}\right|^{2}+(1-\rho) \sigma_{a}^{2}+\sigma_{b}^{2}}}
$$

where $\sigma_{a}^{2}$ and $\sigma_{b}^{2}$ are the variances of AWGN $n_{a}$ and $n_{b}$, respectively.

After amplifying the signal $y_{r}(m)$, the transmitted information of the relay is given by

$$
\begin{aligned}
x_{r}(m) & =\frac{\sqrt{P_{r}} y_{r}(m)}{\sqrt{A}} \\
& =\frac{\sqrt{P_{r}} y_{r}(m)}{\sqrt{(1-\rho) P_{s}\left|h_{S R}\right|^{2}+(1-\rho) \sigma_{a}^{2}+\sigma_{b}^{2}}} .
\end{aligned}
$$

Therefore, the received signal at the destination node, $y_{d}(m)$, is obtained as

$$
y_{d}(m)=h_{R D} \cdot x_{r}(m)+n_{c},
$$


where $h_{R D}=\widetilde{h}_{R D} \sqrt{r^{-\alpha}}, n_{c}$ is the overall AWGN at the destination node, and $\sigma_{c}^{2}$ is the variance of AWGN $n_{c}$. Substituting (7) and (10) into (11), we can get

$$
\begin{aligned}
y_{d}(m)= & \frac{h_{R D} \sqrt{(1-\rho) P_{s} P_{r}} h_{S R} s(m)}{\sqrt{(1-\rho) P_{s}\left|h_{S R}\right|^{2}+(1-\rho) \sigma_{a}^{2}+\sigma_{b}^{2}}} \\
& +\frac{h_{R D} \sqrt{(1-\rho) P_{r}} n_{a}+h_{R D} \sqrt{P_{r}} n_{b}}{\sqrt{(1-\rho) P_{s}\left|h_{S R}\right|^{2}+(1-\rho) \sigma_{a}^{2}+\sigma_{b}^{2}}}+n_{c} \\
= & \frac{h_{R D} \sqrt{(1-\rho) P_{s} P_{r}} h_{S R} s(m)}{\sqrt{(1-\rho) P_{s}\left|h_{S R}\right|^{2}+\sigma_{1}^{2}}} \\
& +\frac{h_{R D} \sqrt{P_{r}} n_{1}}{\sqrt{(1-\rho) P_{s}\left|h_{S R}\right|^{2}+\sigma_{1}^{2}}}+n_{c},
\end{aligned}
$$

where we assume $n_{1}=\sqrt{1-\rho} \cdot n_{a}+n_{b}$ is the total AWGN at the relay and $\sigma_{1}^{2}=(1-\rho) \cdot \sigma_{a}^{2}+\sigma_{b}^{2}$. The first part of (12) is the signal part, and the second and the third part are the noise part. Therefore, the SNR of the $S-R-D$ path at the destination node is obtained as

$\gamma_{S R D}$

$$
\begin{aligned}
& =\frac{\left|h_{R D}\right|^{2}(1-\rho) P_{s} P_{r}\left|h_{S R}\right|^{2} /\left((1-\rho) P_{s}\left|h_{S R}\right|^{2}+\sigma_{1}^{2}\right)}{\left|h_{R D}\right|^{2} P_{r} \sigma_{1}^{2} /\left((1-\rho) P_{s}\left|h_{S R}\right|^{2}+\sigma_{1}^{2}\right)+\sigma_{c}^{2}} \\
& =\frac{\left|h_{R D}\right|^{2}(1-\rho) P_{s} P_{r}\left|h_{S R}\right|^{2}}{\left|h_{R D}\right|^{2} P_{r} \sigma_{1}^{2}+(1-\rho) P_{s}\left|h_{S R}\right|^{2} \sigma_{c}^{2}+\sigma_{1}^{2} \sigma_{c}^{2}} .
\end{aligned}
$$

Substituting (8) into (13), we have

$\gamma_{S R D}$

$$
=\frac{\left|h_{R D}\right|^{2}(1-\rho) P_{s}^{2}\left|h_{S R}\right|^{4} \eta \rho(\tau /(1-\tau))}{\left|h_{R D}\right|^{2}\left|h_{S R}\right|^{2} \eta \rho P_{s} \sigma_{1}^{2}(\tau /(1-\tau))+(1-\rho) P_{s}\left|h_{S R}\right|^{2} \sigma_{c}^{2}+\sigma_{1}^{2} \sigma_{c}^{2}} .
$$

From (2), the probability that the destination decodes the received data packets unsuccessfully, that is, the outage probability, is given by

$$
p_{\text {out_l }}=\mathbb{P}\left(\gamma_{S R D}<\gamma\right)
$$

Then, we have the following theorem.

Theorem 1. The outage probability $p_{\text {out } \_1}$ for $S-R-D$ link with TSPS protocol is obtained as

$$
\begin{aligned}
& p_{\text {out } \_1}=\int_{0}^{d_{2}}(1 \\
& \left.-e^{-\Psi / \Omega \cdot \mu_{S R}} \sqrt{\frac{4 \Lambda r^{\alpha}}{\Omega \cdot \mu_{S R} \mu_{R D}}} K_{1}\left(\sqrt{\frac{4 \Lambda r^{\alpha}}{\Omega \cdot \mu_{S R} \mu_{R D}}}\right)\right) \\
& \cdot \frac{2 r}{d_{2}^{2}} \mathrm{~d} r
\end{aligned}
$$

where $\Lambda=(1-\rho) P_{s} \sigma_{c}^{2} \gamma, \Omega=d_{1}^{-\alpha} P_{s}^{2} \rho(1-\rho) \eta(\tau /(1-\tau))$, and $\Psi=P_{s} \eta \rho \sigma_{1}^{2} \gamma(\tau /(1-\tau)) \cdot \mu_{S R}$ and $\mu_{R D}$ are the mean value of the exponential distributions $\left|\widetilde{h}_{S R}\right|^{2}$ and $\left|\widetilde{h}_{R D}\right|^{2}$, respectively. $K_{1}(\cdot)$ denotes the first-order modified Bessel function of the second kind [16].

Proof. We derive the outage probability $p_{\text {out_1 }}$ by substituting (14) into (15), and $p_{\text {out_l }}$ is given by

$$
\begin{aligned}
p_{\text {out } 1} & =\mathbb{P}\left(\frac{\left|h_{R D}\right|^{2}(1-\rho) P_{s}^{2}\left|h_{S R}\right|^{4} \eta \rho(\tau /(1-\tau))}{\left|h_{R D}\right|^{2}\left|h_{S R}\right|^{2} \eta \rho P_{s} \sigma_{1}^{2}(\tau /(1-\tau))+(1-\rho) P_{s}\left|h_{S R}\right|^{2} \sigma_{c}^{2}+\sigma_{1}^{2} \sigma_{c}^{2}}<\gamma\right) \\
& =\mathbb{P}\left(\left|h_{R D}\right|^{2}<\frac{(1-\rho) P_{s}\left|h_{S R}\right|^{2} \sigma_{c}^{2} \gamma+\sigma_{1}^{2} \sigma_{c}^{2} \gamma}{\left.|| h_{S R}\right|^{4} P_{s}^{2} \rho(1-\rho) \eta(\tau /(1-\tau))-\left|h_{S R}\right|^{2} P_{s} \eta \rho \sigma_{1}^{2} \gamma(\tau /(1-\tau)) \mid}\right) \\
& \stackrel{(a)}{=} \mathbb{P}\left(\left|\widetilde{h}_{R D}\right|^{2}<\frac{(1-\rho) P_{s}\left|\widetilde{h}_{S R}\right|^{2} r^{\alpha} \sigma_{c}^{2} \gamma+\sigma_{1}^{2} \sigma_{c}^{2} \gamma r^{\alpha} d_{1}^{\alpha}}{\left.|| \widetilde{h}_{S R}\right|^{4} d_{1}^{-\alpha} P_{s}^{2} \rho(1-\rho) \eta(\tau /(1-\tau))-\left|\widetilde{h}_{S R}\right|^{2} P_{s} \eta \rho \sigma_{1}^{2} \gamma(\tau /(1-\tau)) \mid}\right) \\
& \stackrel{(b)}{=} \mathbb{P}\left(\left|\widetilde{h}_{R D}\right|^{2}<\frac{\Lambda r^{\alpha}\left|\widetilde{h}_{S R}\right|^{2}+\Gamma}{\left.|\Omega| \widetilde{h}_{S R}\right|^{4}-\Psi\left|\widetilde{h}_{S R}\right|^{2} \mid}\right),
\end{aligned}
$$

where (a) follows from $h_{S R}=\widetilde{h}_{S R} \sqrt{d_{1}^{-\alpha}}$ and $h_{R D}=\widetilde{h}_{R D} \sqrt{r^{-\alpha}}$ and $(b)$ follows from $\Lambda=(1-\rho) P_{s} \sigma_{c}^{2} \gamma, \Gamma=\sigma_{1}^{2} \sigma_{c}^{2} \gamma r^{\alpha} d_{1}^{\alpha}, \Omega=$ $d_{1}^{-\alpha} P_{s}^{2} \rho(1-\rho) \eta(\tau /(1-\tau))$, and $\Psi=P_{s} \eta \rho \sigma_{1}^{2} \gamma(\tau /(1-\tau))$. For simplicity, we consider a high SNR case; the factor $\sigma_{1}^{2} \sigma_{c}^{2}$ in 
the denominator of (14) is approximately 0 , such that $\Gamma \approx 0$. Then, we have

$$
\begin{aligned}
& p_{\text {out_1 }} \approx \mathbb{P}\left(\left|\widetilde{h}_{R D}\right|^{2}<\frac{\Lambda r^{\alpha}\left|\widetilde{h}_{S R}\right|^{2}}{\left.|\Omega| \widetilde{h}_{S R}\right|^{4}-\Psi\left|\widetilde{h}_{S R}\right|^{2} \mid}\right) \\
& =\left\{\begin{array}{l}
\mathbb{P}\left(\left|\widetilde{h}_{R D}\right|^{2}<\frac{\Lambda r^{\alpha}\left|\widetilde{h}_{S R}\right|^{2}}{\Omega\left|\widetilde{h}_{S R}\right|^{4}-\Psi\left|\widetilde{h} h_{S R}\right|^{2}}\right), \quad\left|\widetilde{h}_{S R}\right|^{2}>\frac{\Psi}{\Omega} \\
\mathbb{P}\left(\left|\widetilde{h}_{R D}\right|^{2}>\frac{\Lambda r^{\alpha}\left|\widetilde{h}_{S R}\right|^{2}}{\Omega\left|\widetilde{h}_{S R}\right|^{4}-\Psi\left|\widetilde{h}_{S R}\right|^{2}}\right)=1, \quad\left|\widetilde{h}_{S R}\right|^{2}<\frac{\Psi}{\Omega} .
\end{array}\right.
\end{aligned}
$$

It is worth noting that we have mentioned that $\left|\widetilde{h}_{X Y}\right|^{2} \sim$ $\exp \left(\mu_{X Y}\right)$; the PDF of the exponential variables $\left|\widetilde{h}_{S R}\right|^{2}$ and $\left|\widetilde{h}_{R D}\right|^{2}$ are $f_{\left|\widetilde{h}_{S R}\right|^{2}}(z)=\left(1 / \mu_{S R}\right) e^{-z / \mu_{S R}}$ and $f_{\left|\widetilde{h}_{R D}\right|^{2}}(z)=$ $\left(1 / \mu_{R D}\right) e^{-z / \mu_{R D}}$, respectively. Thus, $p_{\text {out_1 }}$ is approximately given by

$$
\begin{aligned}
& p_{\text {out_1 }}=E_{r}\left[E_{\left|\widetilde{h}_{S R}\right|^{2}}\left[1-e^{-\Lambda r^{\alpha}\left|\widetilde{h}_{S R}\right|^{2} /\left.|\Omega| \widetilde{h}_{S R}\right|^{4}-\Psi\left|\widetilde{h}_{S R}\right|^{2} \mid \cdot 1 / \mu_{R D}}\right]\right] \\
& =E_{r}\left[\int_{0}^{\Psi / \Omega}\left(\frac{1}{\mu_{S R}} e^{-z / \mu_{S R}}\right) \cdot 1 \mathrm{~d} z\right] \\
& +E_{r}\left[\int_{\Psi / \Omega}^{\infty}\left(\frac{1}{\mu_{S R}} e^{-z / \mu_{S R}}\right)\right. \\
& \left.\cdot\left(1-e^{-\left(1 / \mu_{R D}\right)\left(\Lambda r^{\alpha} z /\left(\Omega z^{2}-\Psi z\right)\right)}\right) \mathrm{d} z\right] \\
& \stackrel{(a)}{=} \int_{0}^{d_{2}} \int_{\Psi / \Omega}^{\infty}\left(1-\frac{1}{\mu_{S R}} e^{-\left(z / \mu_{S R}+\Lambda r^{\alpha} z /\left(\Omega z^{2}-\Psi z\right) \cdot 1 / \mu_{R D}\right)}\right) \\
& f(r) \mathrm{d} z \mathrm{~d} r \\
& \stackrel{(b)}{=} \int_{0}^{d_{2}} \int_{\Psi / \Omega}^{\infty}\left(1-\frac{1}{\mu_{S R}} e^{-\left(z / \mu_{S R}+\Lambda r^{\alpha} /(\Omega z-\Psi) \cdot 1 / \mu_{R D}\right)}\right) \\
& \frac{2 r}{d_{2}^{2}} \mathrm{~d} z \mathrm{~d} r \stackrel{(c)}{=} \int_{0}^{d_{2}}(1 \\
& \left.-e^{-\Psi / \Omega \mu_{S R}} \sqrt{\frac{4 \Lambda r^{\alpha}}{\Omega \mu_{S R} \mu_{R D}}} K_{1}\left(\sqrt{\frac{4 \Lambda r^{\alpha}}{\Omega \mu_{S R} \mu_{R D}}}\right)\right) \frac{2 r}{d_{2}^{2}} \mathrm{~d} r,
\end{aligned}
$$

where (a) follows from $0<r \leq d_{2}$, (b) follows from the notion that the PDF of the distance from the relay to the destination is $f(r)=2 r / d_{2}^{2}$, and $(c)$ is obtained by the formula $\int_{0}^{\infty} e^{-\beta / 4 x-\gamma x} \mathrm{~d} x=\sqrt{\beta / \gamma} K_{1}(\sqrt{\beta \gamma})$ [16]. This ends the proof for Theorem 1.

3.2. Direct Link (S-D). As shown in Figure 2, consider the case where the destination node $D$ is located in $\mathscr{B}_{2}$, which is the complementary sector of $\mathscr{B}_{1}$. We have mentioned in Section 2.2 that $l^{2}=d_{1}^{2}+r^{2}-2 d_{1} r \cos \theta$; we have $\mathscr{B}_{2}=\{r \in$ $\left.\left[0, d_{2}\right], \theta \in\left[-\theta_{0}, \theta_{0}\right]\right\}$, with $\theta_{0}=\arccos \left(d_{2} / 2 d_{1}\right)$. Thus, the probability that the destination node is located inside the area $\mathscr{B}_{2}$ is derived as

$$
p_{2}=\frac{1}{2 \pi} \int_{-\theta_{0}}^{\theta_{0}} \mathrm{~d} \theta=\frac{\arccos \left(d_{2} / 2 d_{1}\right)}{\pi} .
$$

Note that $p_{2}$ decreases with $d_{2}$ and increases with $d_{1}$.

In this case, the source transmits towards the destination node directly, and thus the communication is performed within an overall time slot. Therefore, the received signal at the destination node, $y_{d}(t)$, is obtained as

$$
y_{d}(t)=\sqrt{P_{s}} h_{S D} \cdot s(t)+n_{3},
$$

where $h_{S D}=\tilde{h}_{S D} \sqrt{l^{-\alpha}}, n_{3}$ is the AWGN at the destination node, $\sigma_{3}^{2}$ is the variance of AWGN $n_{3}$, and $s(t)$ is normalized transmitted signal from the source. Therefore, the SNR of the $S-D$ path at the destination node is obtained as

$$
\gamma_{S D}=\frac{P_{s}\left|\widetilde{h}_{S D}\right|^{2} l^{-\alpha}}{\sigma_{3}^{2}} .
$$

From (2), the probability that the destination decodes the received data packets unsuccessfully, that is, the outage probability $p_{\text {out_2}}$, is

$$
p_{\text {out } 22}=\mathbb{P}\left(\gamma_{S D}<\gamma\right) \text {. }
$$

Then, we have the following theorem.

Theorem 2. The outage probability $p_{\text {out_2 }}$ for the direct link at the destination node is obtained as

$$
\begin{aligned}
& P_{\text {out } 2} \\
& =\int_{-\arccos \left[d_{2} / 2 d_{1}\right]}^{\arccos \left[d_{2} / 2 d_{1}\right]} \int_{0}^{d_{2}} \frac{r}{\pi d_{2}^{2}} \frac{\sigma_{3}^{2} \gamma\left(d_{1}^{2}+r^{2}-2 d_{1} r \cos [\theta]\right)^{\alpha / 2}}{P_{s} \mu_{S D}} \mathrm{~d} r \mathrm{~d} \theta .
\end{aligned}
$$

Proof. Using (22) in (23), $p_{\text {out_2 }}$ is given by

$$
\begin{aligned}
p_{\text {out } \_2} & =\mathbb{P}\left(\frac{P_{s}\left|\widetilde{h}_{S D}\right|^{2} l^{-\alpha}}{\sigma_{3}^{2}}<\gamma\right) \\
& =\mathbb{P}\left(\left|\widetilde{h}_{S D}\right|^{2}<\frac{\sigma_{3}^{2} \gamma l^{\alpha}}{P_{s}}\right) \\
& \stackrel{(a)}{=} E_{\mathscr{B}_{2}}\left[1-e^{-\sigma_{3}^{2} \gamma l^{\alpha} / P_{s} \mu_{S D}}\right],
\end{aligned}
$$

where (a) follows from the notion that the PDF of the exponential variable $\left|\widetilde{h}_{S D}\right|^{2}$ is $f_{\left|\widetilde{h}_{S D}\right|^{2}}(z)=\left(1 / \mu_{S D}\right) e^{-z / \mu_{S D}}$.

Due to the fact that $l^{2}=d_{1}^{2}+r^{2}-2 d_{1} r \cos \theta$ and $\mathscr{B}_{2}=$ $\left\{r \in\left[0, d_{2}\right], \theta \in\left[-\theta_{0}, \theta_{0}\right]\right\}$, with $\theta_{0}=\arccos \left(d_{2} / 2 d_{1}\right)$, thus, we have

$$
\begin{aligned}
& \text { Pout_2 } \\
& \qquad \int_{-\theta_{0}}^{\theta_{0}} \int_{0}^{d_{2}} \frac{1}{2 \pi}\left(1-e^{-\sigma_{3}^{2} \gamma\left(d_{1}^{2}+r^{2}-2 d_{1} r \cos (\theta)\right)^{\alpha / 2} / P_{s} \mu_{S D}}\right) \\
& \cdot f(r) \mathrm{d} r \mathrm{~d} \theta .
\end{aligned}
$$


For high SNR, we assume $\sigma_{3}^{2} \approx 0$. From the Taylor series $e^{x} \approx 1+x$, the outage probability $p_{\text {out_2 }}$ can be approximately written as

$$
\begin{gathered}
p_{\text {out } 2}=\int_{-\theta_{0}}^{\theta_{0}} \int_{0}^{d_{2}} \frac{1}{2 \pi} \\
\cdot \frac{\sigma_{3}^{2} \gamma\left(d_{1}^{2}+r^{2}-2 d_{1} r \cos (\theta)\right)^{\alpha / 2}}{P_{s} \mu_{S D}} \frac{2 r}{d_{2}^{2}} \mathrm{~d} r \mathrm{~d} \theta .
\end{gathered}
$$

This completes the proof for Theorem 2 .

3.3. Throughput Analysis. For the different locations of the destination node, we have analyzed two conditions for the relay-assisted transmission mode, $S-R-D$ by using the TSPS protocol and direct link $S-D$, and obtained the outage probabilities $p_{\text {out_1 }}$ and $p_{\text {out } 2}$, respectively. The throughput is characterized by evaluating the outage probability at a source transmission rate target, which is defined as $R_{r}=\log (1+\gamma)$. From (3), we denote the throughput with TSPS protocol at the destination node by $C_{a}$; we have

$$
C_{a}=R_{r} \cdot\left(1-p_{\text {out_1 }}\right)(1-\tau) T \text {. }
$$

It is worth noting that, for $\tau \rightarrow 0$ or $\rho \rightarrow 0$, there is less time or less power available for energy harvesting. Consequently, the energy harvested by the relay is smaller and less throughput is obtained due to larger outage probability. On the other hand, for the value of $\tau \rightarrow 1$ or $\rho \rightarrow 1$, there is less time for information transmission or less power portion for data transmission. Furthermore, larger $\rho$ results in poor signal strength at the relay, and when the relay amplifies the noise signal and forwards it to the destination node, small throughput occurs due to larger outage probability and less transmission time. Therefore, there is a tradeoff between energy harvesting and data transmission, and there exist optimal $\tau$ and $\rho$ which yields the maximum network throughput $C_{a}$. by

The throughput for the $S$ - $D$ link denoted by $C_{b}$ is obtained

$$
C_{b}=R_{r}\left(1-p_{\text {out_2 }}\right) T \text {. }
$$

Therefore, the throughput for the relay-assisted transmission mode $C_{1}$, at the destination node, is obtained by

$$
\begin{aligned}
C_{1}= & C_{a} \cdot p_{1}+C_{b} \cdot p_{2} \\
= & R_{r} \cdot\left(1-p_{\text {out_1 }}\right)(1-\tau) T \cdot p_{1} \\
& +R_{r}\left(1-p_{\text {out_2 }}\right) T \cdot p_{2},
\end{aligned}
$$

where $p_{1}$ and $p_{2}$ are given by (4) and (20), respectively. It is worth highlighting that $C_{b}$ is a constant for different $\tau$ and $\rho$; consequently, the optimal value of $C_{1}$ mainly depends on $C_{a}$.

It seems intractable to evaluate the closed-form expressions for the optimal value of $C_{1}$. However, the optimization can be done offline by numerical calculation of the optimal values of $\rho^{*}$ and $\tau^{*}$ for the given system parameters.

\section{Nonrelay Mode}

In this section, we will characterize the outage probability and the network throughput for the link from source to the destination. As shown in Figure 2, consider the case where the destination node $D$ is located inside the area $\mathscr{B}$, and the communication is performed in an overall time slot. Similar to that in Section 3.2, the SNR of the nonrelay mode at the destination node can be obtained as

$$
\gamma_{S D}=\frac{P_{s}\left|\tilde{h}_{S D}\right|^{2} l^{-\alpha}}{\sigma_{4}^{2}},
$$

where $\sigma_{4}^{2}$ is the variance of the overall AWGN at the destination node. From (2), the probability that the destination node decodes the received data packets unsuccessfully, that is, the outage probability $p_{\text {out_3 } 3}$, is

$$
p_{\text {out } 3}=\mathbb{P}\left(\gamma_{S D}<\gamma\right) \text {. }
$$

Then, we have the following theorem.

Theorem 3. The outage probability $p_{\text {out_3 } 3}$ for the nonrelay mode at the destination node is obtained as

$$
\begin{aligned}
& P_{\text {out } 33} \\
& =\int_{-\pi}^{\pi} \int_{0}^{d_{2}} \frac{\sigma_{4}^{2} \gamma\left(d_{1}^{2}+r^{2}-2 d_{1} r \cos (\delta)\right)^{\alpha / 2}}{P_{s} \mu_{S D}} \frac{r}{\pi d_{2}^{2}} \mathrm{~d} r \mathrm{~d} \delta .
\end{aligned}
$$

Proof. Substituting (31) into (32), $p_{\text {out_3 }}$ is given by

$$
\begin{aligned}
p_{\text {out_3 }} & =\mathbb{P}\left(\frac{P_{s}\left|\tilde{h}_{S D}\right|^{2} l^{-\alpha}}{\sigma_{4}^{2}}<\gamma\right) \\
& =E_{\mathscr{B}}\left[1-e^{-\sigma_{4}^{2} \gamma l^{\alpha} / P_{s} \mu_{S D}}\right] .
\end{aligned}
$$

From Section 2.2, we get that $\mathscr{B}=\left\{r \in\left[0, d_{2}\right], \delta \in\right.$ $[-\pi, \pi]\}$. Thus, we have

$$
\begin{aligned}
& P_{\text {out_3 }} \\
& \qquad \int_{-\pi}^{\pi} \int_{0}^{d_{2}}\left(1-e^{-\sigma_{4}^{2} \gamma\left(d_{1}^{2}+r^{2}-2 d_{1} r \cos (\delta)\right)^{\alpha / 2} / P_{s} \mu_{S D}}\right) \frac{1}{2 \pi} \\
& \cdot f(r) \mathrm{d} r \mathrm{~d} \delta .
\end{aligned}
$$

Similarly, from the Taylor series $e^{x} \approx 1+x$, the outage probability $p_{\text {out_3 }}$ can be approximately written as

$$
\begin{gathered}
p_{\text {out } 33}=\int_{-\pi}^{\pi} \int_{0}^{d_{2}} \frac{\sigma_{4}^{2} \gamma\left(d_{1}^{2}+r^{2}-2 d_{1} r \cos (\delta)\right)^{\alpha / 2}}{P_{s} \mu_{S D}} \frac{1}{2 \pi} \\
\cdot \frac{2 r}{d_{2}^{2}} \mathrm{~d} r \mathrm{~d} \delta .
\end{gathered}
$$

This completes the proof for Theorem 3.

The throughput $C_{2}$ at the destination node in the nonrelay transmission mode is obtained by

$$
C_{2}=R_{r} \cdot\left(1-p_{\text {out_3 }}\right) T \text {. }
$$




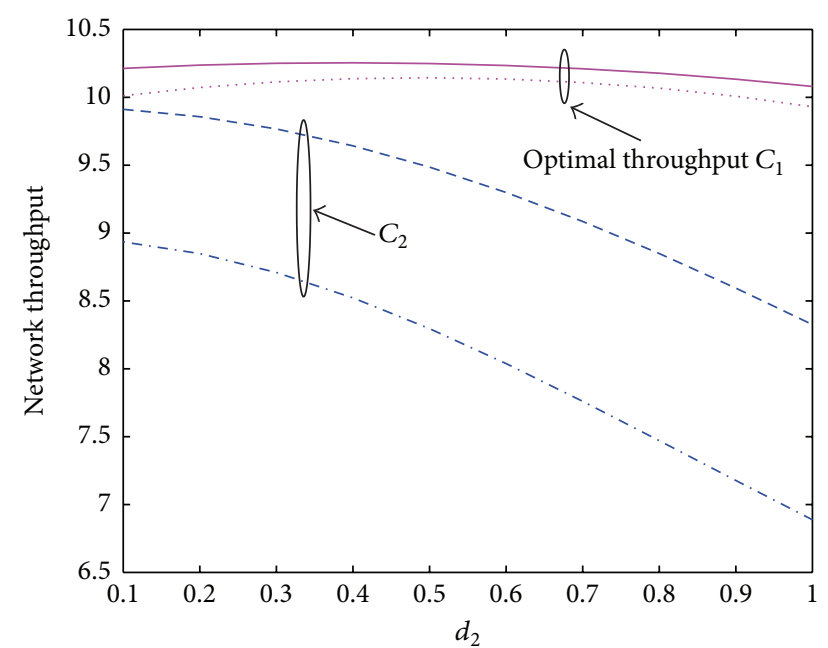

Relay-assisted mode, with $P_{s}=1$

...- Nonrelay mode, with $P_{s}=1$

— Relay-assisted mode, with $P_{s}=2$

- - Nonrelay mode, with $P_{s}=2$

FIgURE 4: The network throughput $C$ of the two transmission modes versus the transmission rate target $R_{r}$, with $\sigma_{1}^{2}=0.01$ and $\sigma_{c}^{2}=$ 0.001 .

\section{Numerical Results}

In this section, based on our theoretical analysis, we present some numerical results and give some interpretations. First of all, we compare the optimal throughput in relay-assisted transmission mode with that in nonrelay mode. By using the TSPS protocol, we characterize the optimal values $C_{1}$ of the network throughput in relay-assisted mode, the optimal values of the time switching ratio $\tau$, and the power splitting ratio $\rho$ for different parameters, respectively.

Unless otherwise specified, we set the path-loss exponent as $\alpha=4$, which corresponds to a city cellular network environment. Transmission time slot is normalized to $T=$ 1 ; the energy harvesting efficiency $\eta=1$; the source transmission power $P_{s}=1 \mathrm{~W}$; the source transmission rate target $R_{r}=11 \mathrm{bits} / \mathrm{s} / \mathrm{Hz}$; the distance between the source node and the relay and the radius of the selection sector are both normalized to $d_{1}=d_{2}=1$; and the mean value of the channel power gain $\mu_{S R}=\mu_{R D}=\mu_{S D}=1$.

5.1. Comparison between the Relay-Assisted Transmission Mode and the Nonrelay Mode. Figure 4 shows the optimal throughput in the relay-assisted transmission mode and the network throughput in the nonrelay mode for different values of the transmission rate target $R_{r}$. We have the following observations. First of all, the optimal throughput $C_{1}$ in the relay-assisted transmission mode renders larger values of throughput compared to the throughput in the nonrelay mode $C_{2}$, as much as $30.8 \%$, when the rate target is 11.5 at $P_{s}=$ 0.3 . Moreover, the network throughput first increases as $R_{r}$ increases but starts to decrease when the rate target is above a certain threshold. This is due to the fact that, for smaller $R_{r}$, the network throughput mainly depends on the rate target $R_{r}$,

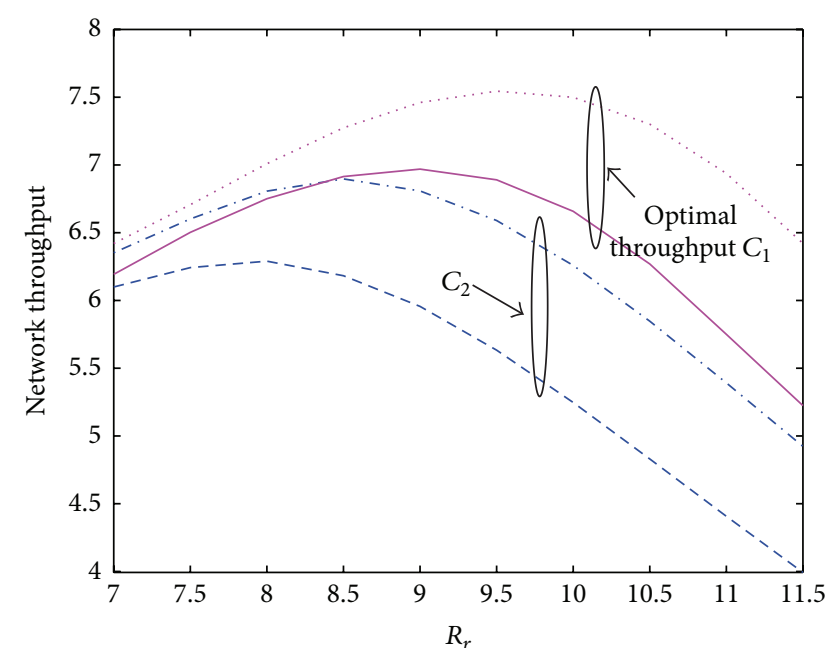

Relay-assisted mode, with $P_{s}=0.5$

. . - Nonrelay mode, with $P_{s}=0.5$

- Relay-assisted mode, with $P_{s}=0.3$

- - Nonrelay mode, with $P_{s}=0.3$

FIGURE 5: The network throughput $C$ of the two transmission modes versus the radius of selection sector $d_{2}$, with $\sigma_{1}^{2}=\sigma_{c}^{2}=0.001$.

and the throughput increases as $R_{r}$ increases. However, when $R_{r}$ increases above a certain value, the destination node fails to decode the signal correctly, which results in a larger outage probability, and thus the throughput decreases. Furthermore, it is observed that the throughput increases with increasing $P_{s}$; the reason is that large source transmission power $P_{s}$ is beneficial to reducing the network outage probability and thus enhances the network throughput.

Figure 5 shows the optimal throughput in the relayassisted transmission mode and the network throughput in the nonrelay mode for different values of the radius of selection sector $d_{2}$. Similar to Figure 4 , the optimal throughput in the relay-assisted transmission mode, $C_{1}$, renders larger values of throughput compared to the throughput in the nonrelay mode, $C_{2}$, as much as $44.2 \%$, when $d_{2}=1$ at $P_{s}=1$, and the throughput increases with increasing $P_{s}$. Moreover, the throughput in the nonrelay mode $C_{2}$ decreases as $d_{2}$ increases due to the fact that the path loss increases with increasing $d_{2}$ and thus results in a large outage probability $p_{\text {out_3 }}$ (see (37)). However, the optimal throughput in the relay-assisted transmission mode $C_{1}$ is not monotonously changed with $d_{2}$; this is because $d_{2}$ mainly affects the throughput in relay-assisted transmission mode with TSPS protocol (see the first portion of (30)). On the one hand, small $d_{2}$ leads to a small path loss, which imposes a small outage probability $p_{\text {out_1 }}$, and thus results in large throughput $C_{a}$ (see (28)), but the probability $p_{1}$ from (4) is decreased. On the other hand, for large values of $d_{2}$, the throughput $C_{a}$ degrades since the outage probability $p_{\text {out_1 }}$ increases, but the probability $p_{1}$ is increased. Therefore, there exists an optimal $d_{2}$ which yields the maximum throughput in the relay-assisted transmission mode $C_{1}$. In Figure 5, the optimal $d_{2}$ is 0.4 and 0.5 , with $P_{s}=2$ and $P_{s}=1$, respectively. 


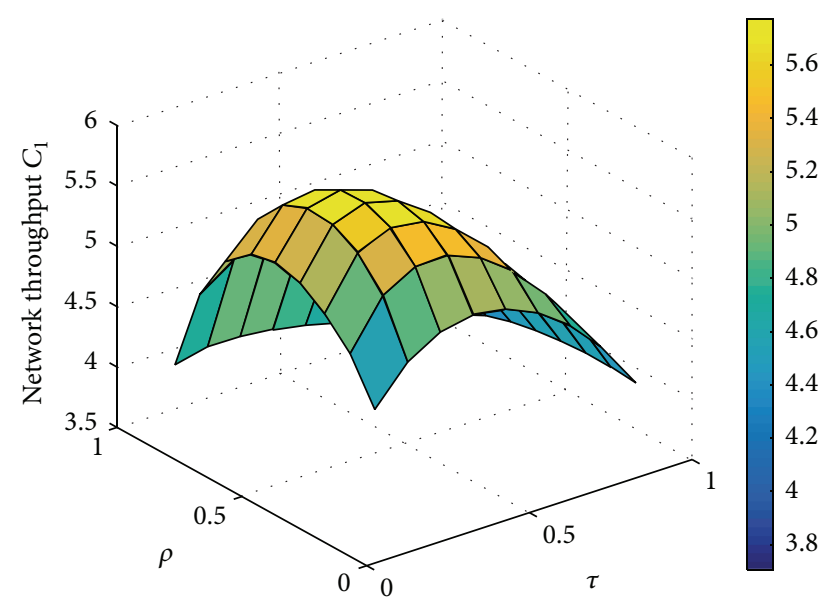

FIgURE 6: The network throughput $C_{1}$ with different settings of the time switching ratio $\tau$ and the power splitting ratio $\rho$ in the relayassisted mode, with $\sigma_{1}^{2}=\sigma_{c}^{2}=0.01$.

5.2. Optimization with the Relay-Assisted Transmission Mode. Figure 6 demonstrates how the time switching ratio $\tau$ and the power splitting ratio $\rho$ would affect the network throughput $C_{1}$. As observed, the maximum $C_{1}$ can be obtained as $C_{1}=$ 5.7752 with $\tau=0.3$ and $\rho=0.4$. This is because, for small values of $\tau$ or $\rho$, there is less time and there is less power available for energy harvesting. Consequently, the energy harvested by the relay is smaller and less throughput is obtained due to larger outage probability. On the other hand, for the value of $\tau$ or $\rho$ larger than the optimal values, there is less time for information transmission and there is less power portion for data transmission. Moreover, larger $\rho$ results in poor signal strength at the relay, and when the relay amplifies and transmits the noise signal to the destination node, small throughput occurs due to larger outage probability at the destination node. Our results can be used to find the feasible region in the $\tau$ - $\rho$ plane for given allowable throughput. Figure 7 demonstrates the network throughput for TSPS protocol with $0<\rho<1$. We see that the simulation results are consistent with our analytical results for the different values of $\rho$ and $\tau$, which verifies the analytical expression for the network throughput.

Figure 8 shows the optimal throughput $C_{1}$ with relayassisted transmission protocol as well as the TSR and the PSR protocols for different values of energy harvesting efficiency $\eta$. It is observed that the optimal throughput $C_{1}$ is in proportion to energy harvesting efficiency $\eta$. Furthermore, the TSPS protocol outperforms the TSR and the PSR protocols in terms of throughput, as much as $5.9 \%$ and $28 \%$, respectively, when the energy harvesting efficiency $=1$. Figure 9 plots the optimal throughput $C_{1}$ with relay-assisted transmission protocol as well as the TSR and the PSR protocols for different values of the noise variance for the $R-D \operatorname{link} \sigma_{c}^{2}$. It is observed that the optimal throughput $C_{1}$ is inversely proportional to the noise variance for the $R-D$ link $\sigma_{c}^{2}$. Similarly, the TSPS protocol outperforms the TSR and the PSR protocols in terms of throughput, as much as $7.3 \%$ and $28 \%$, respectively, when the noise variance for the $R-D$ link $\sigma_{c}^{2}=10^{-3}$. We can see

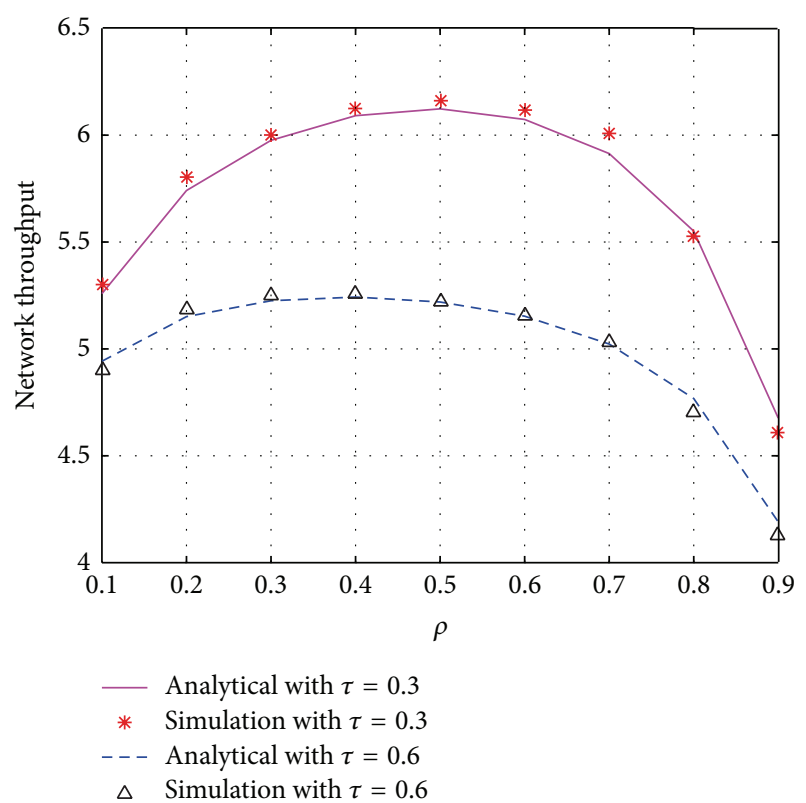

FIgURE 7: The network throughput $C_{1}$ with different settings of the power splitting ratio $\rho$ in the relay-assisted mode.

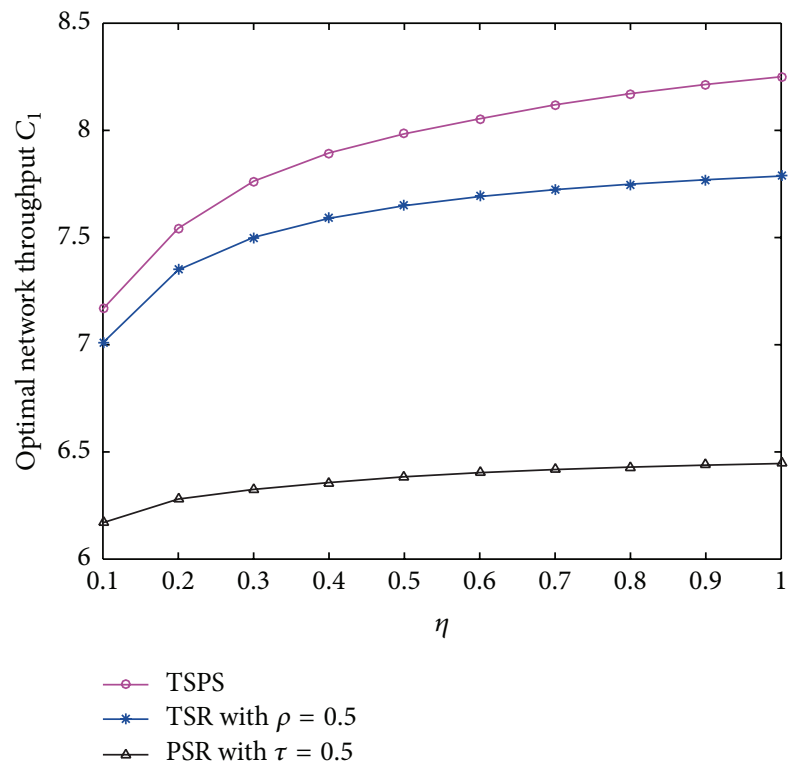

FIgURE 8: The optimal network throughput $C_{1}$ with relay-assisted transmission mode versus the energy harvesting efficiency $\eta$, with $\sigma_{1}^{2}=0.01$ and $\sigma_{c}^{2}=0.001$.

that joint optimization of both $\rho$ and $\tau$ improves the network throughput significantly.

In Figure 10, we characterized the optimal values of $\tau$ and $\rho$ for the TSPS protocol for different values of the noise variance for the $R-D$ link $\sigma_{c}^{2}$. It can be observed from Figure 10 that the optimal value of $\tau$ or $\rho$ increases by increasing $\sigma_{c}^{2}$, and the optimal values of $\tau$ and $\rho$ jointly maximize the network throughput as shown in Figure 9 with TSPS protocol. 


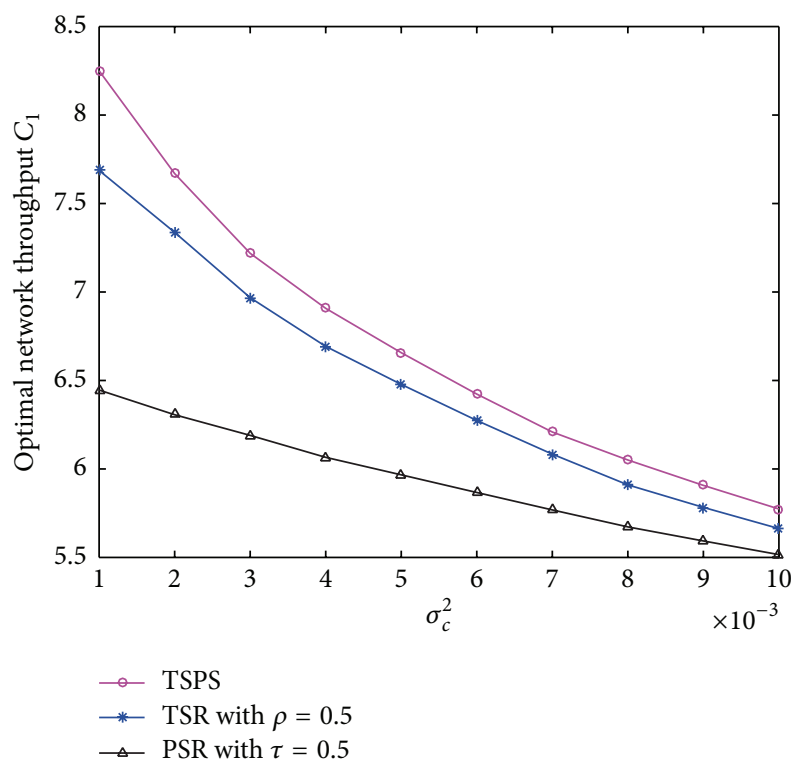

FIGURE 9: The optimal network throughput $C_{1}$ with relay-assisted transmission mode versus the noise variance for the $R-D$ link $\sigma_{c}^{2}$, with $\sigma_{1}^{2}=0.01$.

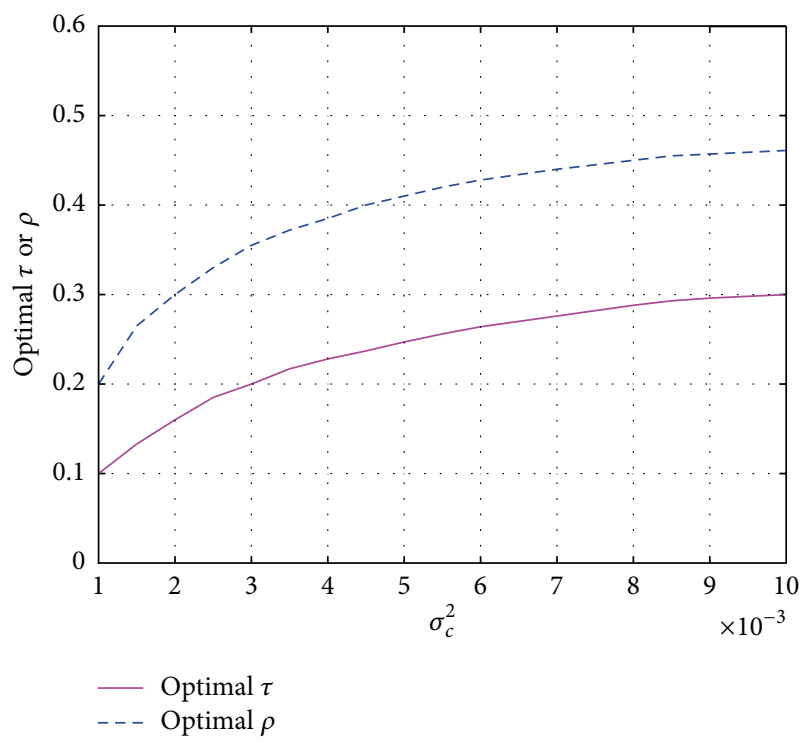

FIGURE 10: The optimal time switching ratio $\tau$ and power splitting ratio $\rho$ versus the noise variance for the $R-D$ link $\sigma_{c}^{2}$, with $\sigma_{1}^{2}=0.01$.

\section{Conclusion}

In this paper, we investigated two transmission modes: the relay-assisted transmission mode and the nonrelay mode, respectively. In the relay-assisted transmission mode, we proposed a TSPS protocol in a cooperative relaying system to improve network performance in terms of efficiency and reliability. During the first amount of the time slot, a portion of the received signal power is used for energy harvesting by the relay, and the remaining power is used for information transmission from the source to the relay, while the remaining part of the time is used for information transfer from the relay to the destination. The outage probability and the network throughput are characterized, by using the two transmission modes, respectively. With the TSPS protocol, the network throughput is maximized by joint optimization of the power splitting and time switching ratios. In particular, theoretical analysis shows that the optimal throughput in the relay-assisted transmission mode achieves larger values of throughput compared to the throughput in the nonrelay mode as much as $44.2 \%$.

\section{Symbol Notation}

$\gamma: \quad$ The signal-to-noise (SNR) target

$R_{r}$ : The transmission rate target

$\tau: \quad$ Time switching ratio

$\rho: \quad$ Power splitting ratio

$P_{s}$ : Transmission power of the source

$h$ : Rayleigh fading gain

$p_{\text {out }}$ : Outage probability of the network

$C: \quad$ Network throughput

$d_{1}$ : The fixed distance between the source and the relay

$d_{2}$ : The radius of the available region of the relay

$\mathscr{B}_{1}$ : Selection sector of the relaying path

$l$ : The distance between the source and the destination node

$r$ : The distance between the relay and the destination node

$\eta$ : $\quad$ Energy harvesting efficiency

$\alpha$ : Path-loss exponent

$T$ : One transmission slot time

$\sigma_{1}^{2}$ : The variance of the additive white Gaussian noise (AWGN) over the $S$ - $R$ link

$\sigma_{c}^{2}$ : The variance of the AWGN over the $R-D$ link.

\section{Competing Interests}

The authors declare that they have no competing interests.

\section{Acknowledgments}

This work was supported in part by the National Science and Technology Major Project under Grant 2015ZX03003012005, the NSFC under Grant 61271257, and the National Research Foundation for the Doctoral Program of Higher Education of China under Grant 20120005110007.

\section{References}

[1] H. Chen, Y. Li, J. L. Rebelatto, B. F. Uchôa-Filho, and B. Vucetic, "Harvest-then-cooperate: wireless-powered cooperative communications," IEEE Transactions on Signal Processing, vol. 63, no. 7, pp. 1700-1711, 2015.

[2] H. Li, J. Xu, R. Zhang, and S. Cui, "A general utility optimization framework for energy-harvesting-based wireless communications," IEEE Communications Magazine, vol. 53, no. 4, pp. 7985, 2015. 
[3] A. Sinha and A. Chandrakasan, "Dynamic power management in wireless sensor networks," IEEE Design and Test of Computers, vol. 18, no. 2, pp. 62-74, 2001.

[4] D. Bouchouicha, F. Dupont, M. Latrach, and L. Ventura, "Ambient RF energy harvesting," in Proceedings of the International Conference on Renewable Energies and Power Quality (ICREPQ '10), Granada, Spain, March 2010.

[5] A. M. Zungeru, L.-M. Ang, S. R. S. Prabaharan, and K. P. Seng, "Radio frequency energy harvesting and management for wireless sensor networks," in Green Mobile Devices and Networks: Energy Optimization and Scavenging Techniques, $\mathrm{H}$. Venkataraman and G.-M. Muntean, Eds., chapter 13, pp. 341368, CRC Press, New York, NY, USA, 2012.

[6] S. Lee, R. Zhang, and K. Huang, "Opportunistic wireless energy harvesting in cognitive radio networks," IEEE Transactions on Wireless Communications, vol. 12, no. 9, pp. 4788-4799, 2013.

[7] X. Lu, P. Wang, D. Niyato, D. I. Kim, and Z. Han, "Wireless networks with rf energy harvesting: a contemporary survey," IEEE Communications Surveys and Tutorials, vol. 17, no. 2, pp. 757-789, 2015.

[8] L. R. Varshney, "Transporting information and energy simultaneously," in Proceedings of the IEEE International Symposium on Information Theory (ISIT '08), pp. 1612-1616, Toronto, Canada, July 2008.

[9] X. Zhou, R. Zhang, and C. K. Ho, "Wireless information and power transfer: architecture design and rate-energy tradeoff," IEEE Transactions on Communications, vol. 61, no. 11, pp. 47544767, 2013.

[10] L. Liu, R. Zhang, and K.-C. Chua, "Wireless information transfer with opportunistic energy harvesting," IEEE Transactions on Wireless Communications, vol. 12, no. 1, pp. 288-300, 2013.

[11] D. W. K. Ng, E. S. Lo, and R. Schober, "Wireless information and power transfer: energy efficiency optimization in OFDMA systems," IEEE Transactions on Wireless Communications, vol. 12, no. 12, pp. 6352-6370, 2013.

[12] A. A. Nasir, X. Zhou, S. Durrani, and R. A. Kennedy, "Relaying protocols for wireless energy harvesting and information processing," IEEE Transactions on Wireless Communications, vol.12, no. 7, pp. 3622-3636, 2013.

[13] Y. Liu and X. Wang, "Information and energy cooperation in OFDM relaying," in Proceedings of the IEEE ICC, London, UK, June 2015.

[14] M. O. Hasna and M.-S. Alouini, "Performance analysis of twohop relayed transmissions over Rayleigh fading channels," in Proceedings of the IEEE 56th Vehicular Technology Conference (VTC-Fall '02), vol. 4, pp. 1992-1996, Vancouver, Canada, 2002.

[15] S. Ikki and M. H. Ahmed, "Performance analysis of cooperative diversity wireless networks over Nakagami-m fading channel," IEEE Communications Letters, vol. 11, no. 4, pp. 334-336, 2007.

[16] I. S. Gradshteyn and I. M. Ryzhik, Table of Integrals, Series, and Products, Academic Press, New York, NY, USA, 4th edition, 1980. 

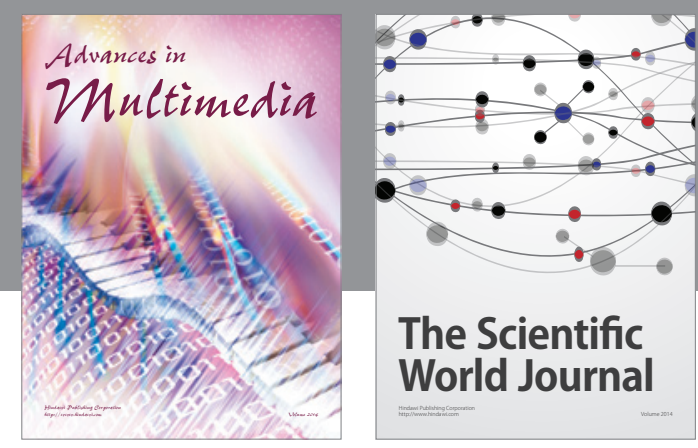

The Scientific World Journal
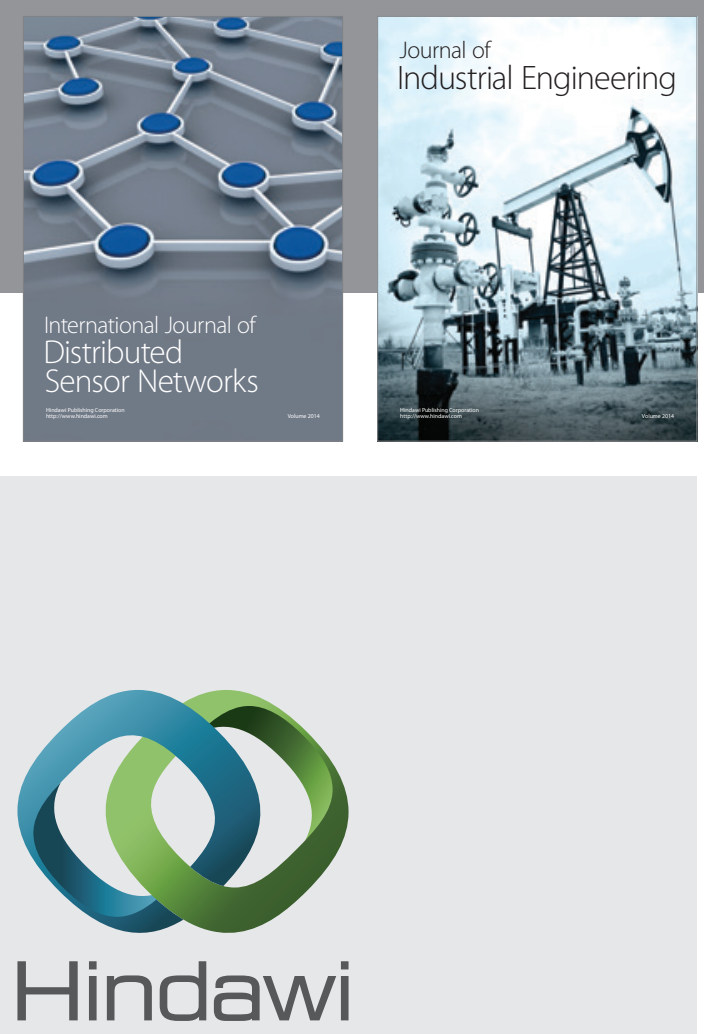

Submit your manuscripts at

http://www.hindawi.com

\section{Computer Networks} and Communications
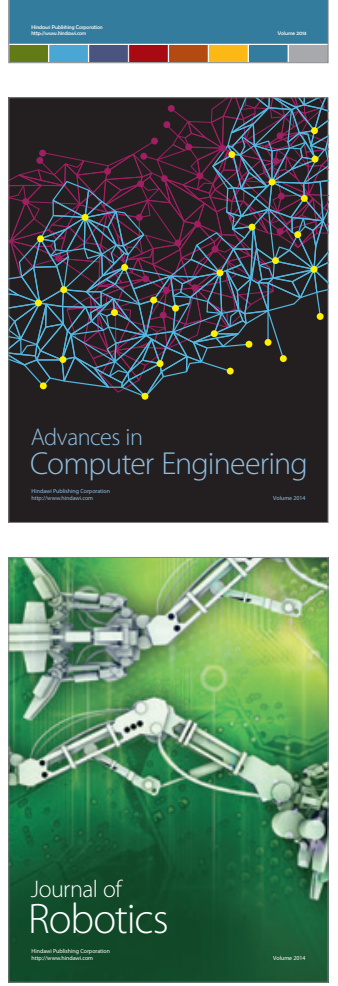
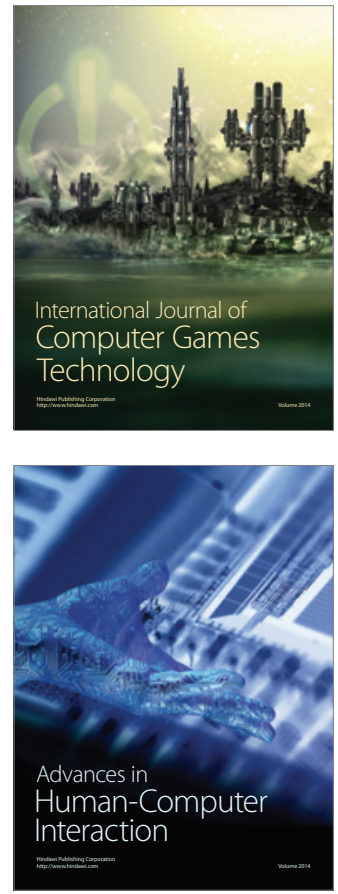
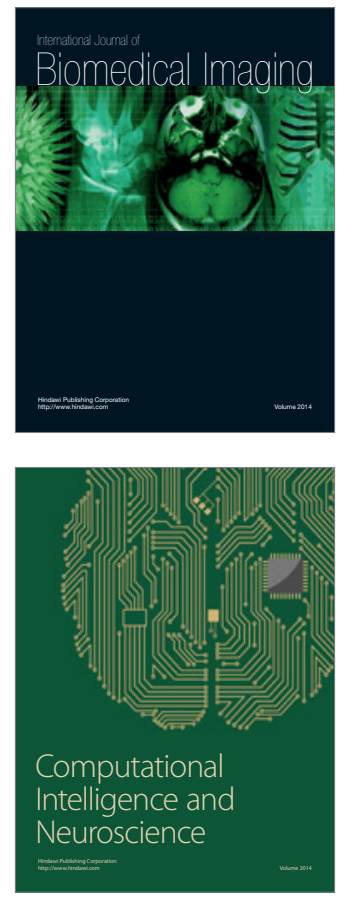
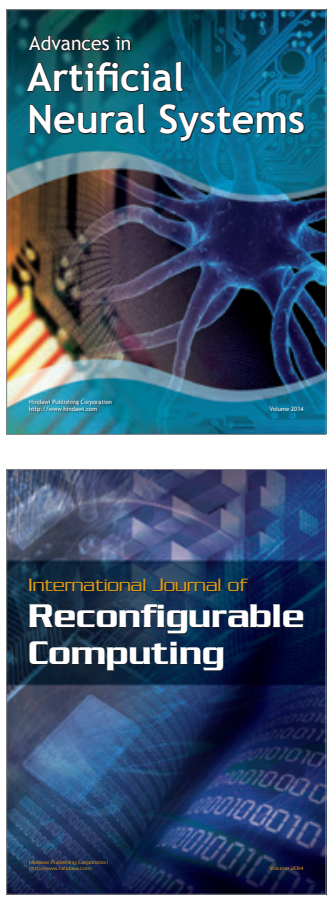
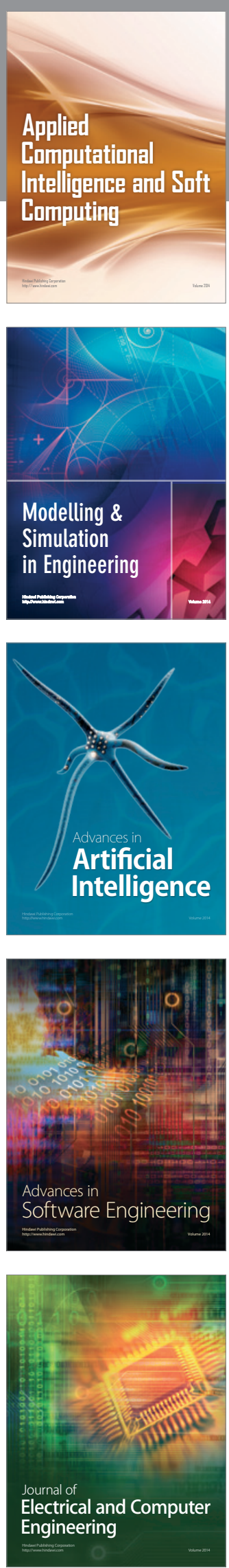